\title{
Corrigendum to "Evaluation of the Correlation between Serum Concentrations of Asymmetric Dimethylarginine and Corrected TIMI Frame Count in Patients with Slow Coronary Flow"
}

\author{
Mahshid Naserifar, ${ }^{1}$ Mahshid Ataei, ${ }^{1}$ Nadia Behzadian, ${ }^{1}$ \\ Amir Hooshang Mohammadpour, ${ }^{1,2}$ Mostafa Dastani, ${ }^{3}$ Jamal Shamsara $\mathbb{D}^{,}{ }^{2}$ Sepideh Elyasi, \\ Amirhossein Sahebkar $\mathbb{D}^{4,5,6}$ and Hesamoddin Hosseinjani ${ }^{1}{ }^{1}$ \\ ${ }^{1}$ Department of Clinical Pharmacy, School of Pharmacy, Mashhad University of Medical Sciences, Mashhad, Iran \\ ${ }^{2}$ Pharmaceutical Research Center, Pharmaceutical Technology Institute, Mashhad University of Medical Sciences, Mashhad, Iran \\ ${ }^{3}$ Department of Cardiology, School of Medicine, Mashhad University of Medical Sciences, Mashhad, Iran \\ ${ }^{4}$ Biotechnology Research Center, Pharmaceutical Technology Institute, Mashhad University of Medical Sciences, Mashhad, Iran \\ ${ }^{5}$ Neurogenic Inflammation Research Center, Mashhad University of Medical Sciences, Mashhad, Iran \\ ${ }^{6}$ Polish Mother's Memorial Hospital Research Institute (PMMHRI), Lodz, Poland
}

Correspondence should be addressed to Hesamoddin Hosseinjani; hosseinjanih@mums.ac.ir

Received 5 December 2020; Accepted 5 December 2020; Published 14 December 2020

Copyright (C) 2020 Mahshid Naserifar et al. This is an open access article distributed under the Creative Commons Attribution License, which permits unrestricted use, distribution, and reproduction in any medium, provided the original work is properly cited.

In the article titled "Evaluation of the Correlation between Serum Concentrations of Asymmetric Dimethylarginine and Corrected TIMI Frame Count in Patients with Slow Coronary Flow" [1], Dr. Jamal Shamsara and Dr. Sepideh Elyasi were missing from the author list. Dr. Elyasi contributed to the study planning, data analysis, and manuscript preparation. Dr. Shamsara contributed to the study design, data analysis, and manuscript preparation. The corrected author list is shown above.

\section{References}

[1] M. Naserifar, M. Ataei, N. Behzadian et al., "Evaluation of the correlation between serum concentrations of asymmetric dimethylarginine and corrected TIMI frame count in patients with slow coronary flow," International Journal of Vascular Medicine, vol. 2020, Article ID 4592190, 6 pages, 2020. 